\section{Anharmonic Effects on Charge and Orbital Order}

In a recent Letter [1], Mutou and Kontani tried to explain the experimentally observed charge and orbital order (COO) in half-doped manganites (upper left corner in Fig. 1). Some theories for the COO rely on the magnetic CE-type structure as an important ingredient [2]. Experimentally, however, at finite temperatures the $\mathrm{COO}$ is also observed without magnetic $\mathrm{CE}$ order. It is therefore desirable to obtain the $\mathrm{COO}$ without relying on magnetic interactions. Because of incorrect assumptions made in hopping integrals, however, the explanation in Ref. [1] is not valid. We demonstrate this below and show that the observed $\mathrm{COO}$ can be stabilized by anharmonic effects which always favor local elongation of oxygen octahedra around Jahn-Teller ions. The ratios of the hopping integrals $t_{i j}^{\alpha \beta}$ in the Hamiltonian $H=\sum_{\langle i j\rangle} t_{i j}^{\alpha \beta} c_{i \alpha}^{\dagger} c_{j \beta}+$ $U \sum_{i,(\alpha \neq \beta)} n_{i \alpha} n_{i \beta}+V \sum_{\langle i j\rangle} n_{i} n_{j}$, between orbital $\alpha$ on the site $i$ and $\beta$ on $j$ [1], are known and related by symmetry $\left(\alpha, \beta=z^{2}=3 z^{2}-r^{2}, x^{2}-y^{2}\right)$. If we take $t_{\langle i j\rangle \| z}^{z^{2}, z^{2}}=t$, then $t_{\langle i j\rangle \| x, y}^{z^{2}, z^{2}}=t / 4, t_{\langle i j\rangle \| x, y}^{z^{2}, x^{2}-y^{2}}= \pm \frac{\sqrt{3}}{2} t$, etc. In particular, the hopping integral $\frac{x^{2}, y^{2}}{\langle i j\rangle \| x, y}=t / 2$, and not zero as is assumed in Ref. [1] (here orbitals $\left.x^{2}=3 x^{2}-r^{2}, y^{2}=3 y^{2}-r^{2}\right)$. This incorrect simplification has important consequences. If we take the correct values of the hopping integrals, the lowest energy state is not the experimentally observed COO, but the state where $\left(x^{2}-y^{2}\right)$ orbitals are occupied at the $\mathrm{Mn}^{3+}$-sites. In Fig. 1 we show the energies of both states, calculated in a mean-field approximation, for $U / t=8$. One can easily understand this result by comparing energies for $U, V \gg t: E_{\mathrm{COO}}=-\frac{5}{6} t^{2} / V$ and $E_{x^{2}-y^{2}}=-t^{2} / V$. Thus the conclusion of Ref. [1] that their model reproduces the experimentally observed $\mathrm{COO}$ at $x=1 / 2$ is based on wrong approximations and is incorrect.

One can however obtain the correct $\mathrm{COO}$ without appealing to magnetic interactions. The occupation of the $x^{2}-y^{2}$ orbitals corresponds to locally compressed octahedra and is unfavorable due to the anharmonic effects which always stabilize elongated octahedra. The physical reasons for this are rather simple: concomitant with the occupation of a particular orbital, the local surrounding of the Jahn-Teller ion distorts. The Mn-O distances $a$, $b$, and $c$ change to $a \rightarrow a_{0}+u_{a}, b \rightarrow b_{0}+u_{b}$, and

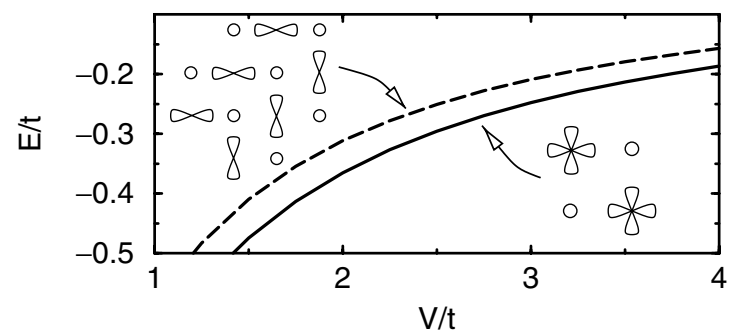

FIG. 1. Mean-field energy of the experimentally observed COO (dashed line) and the $x^{2}-y^{2}$ state (full line). $c \rightarrow c_{0}+u_{c}$. For an occupied $z^{2}$ orbital we have elongation along the $c$ axis, $u_{c}=2 \delta$, and a contraction in the other directions $u_{a}=u_{b}=-\delta, \delta>0$. If the $x^{2}-y^{2}$ is occupied we have a similar distortion with $\delta<0$. The loss of elastic energy at such a distortion consists of harmonic and anharmonic contributions, $\sum_{l} \frac{B}{2} u_{l}^{2}-\frac{\zeta}{3} u_{l}^{3}$, with the coefficient $\zeta>0$ and the sum over the three directions. One immediately sees that the harmonic terms do not discriminate between different orbitals: all states $|\theta\rangle=\cos \frac{\theta}{2}\left|z^{2}\right\rangle+\sin \frac{\theta}{2}\left|x^{2}-y^{2}\right\rangle$ have the same energy. However, the anharmonic term always stabilizes local elongation $\delta>0$ (which may be directed along $x, y$, or $z$ directions). This can be modeled by adding the term $E_{\text {anh }}=-\zeta \cos 3 \theta$ to the energy [3]. For large enough $\zeta$ only the states $\left|z^{2}\right\rangle,\left|x^{2}\right\rangle$, or $\left|y^{2}\right\rangle\left(\theta=0, \pm \frac{2 \pi}{3}\right)$ can be occupied. This anharmonic contribution has very general character; it is most probably the reason that among hundreds of known Jahn-Teller systems with localized electrons none has locally compressed octahedra. If the anharmonic term is taken into account states with occupied $x^{2}$ and/or $y^{2}$ orbitals become preferred and the state with $\left(x^{2}-y^{2}\right)$ occupation is destabilized with increasing $\zeta, E_{x^{2}-y^{2}}=E_{x^{2}-y^{2}}^{o}+\zeta, E_{\mathrm{COO}}=E_{\mathrm{COO}}^{o}-\zeta$, so that the experimental COO is lower in energy for $\zeta>t^{2} /(12 \mathrm{~V})$. The choice between different $\mathrm{OO}$ states made of elongated orbitals is, however, a delicate problem: besides the experimentally observed $\mathrm{COO}$ state, one has to consider, for instance, also the state where on all $\mathrm{Mn}^{3+}$ sites the $x^{2}$ orbitals are occupied. Using the same arguments as in Ref. [1], it is easy to show that the observed COO state is stable at least for $U, V \gg t$.

Thus one can indeed obtain the correct $\mathrm{COO}$ in halfdoped manganites without invoking magnetic interactions, but only if one includes the strong tendency to stabilize the locally elongated octahedra around localized Jahn-Teller ions. This factor definitely plays an important role also in other situations, e.g., for the stripe phases in the overdoped regime.

Daniel Khomskii ${ }^{1}$ and Jeroen van den Brink ${ }^{2}$

${ }^{1}$ Laboratory of Applied and Solid State Physics

University of Groningen

Nijenborgh 4, 9747 AG Groningen, The Netherlands

${ }^{2}$ Faculty of Applied Physics

University of Twente

P.O. Box 217, 7500 AE Enschede, The Netherlands

Received 4 April 2000

PACS numbers: 71.20.Be

[1] T. Mutou and H. Kontani, Phys. Rev. Lett. 83, 3685 (1999).

[2] I. V. Solovyev and K. Terakura, Phys. Rev. Lett. 83, 2825 (1999); J. van den Brink, G. Khaliullin, and D. Khomskii, Phys. Rev. Lett. 83, 5118 (1999); S. Yunoki, T. Hotta, and E. Dagotto, Phys. Rev. Lett. 84, 3714 (2000).

[3] J. Kanamori, J. Appl. Phys. 31, 14S (1960).

(C) 2000 The American Physical Society

3329 